\title{
PRE-SUMBISSION VERSION
}

This is a pre-review version of the manuscript:

Strickland, M. A., C. T. Arsene, et al. (2010). "A multi-platform comparison of efficient probabilistic methods in the prediction of total knee replacement mechanics." Comput Methods Biomech Biomed Engin 13(6): 701-709.

The full, final, peer-reviewed and proofed version is available from the publisher:

http://dx.doi.org/10.1080/10255840903476463 


\section{A Multi-Platform Comparison of Efficient Probabilistic Methods in the Prediction of Total Knee Replacement Mechanics}

M. A. Strickland ${ }^{1}$, C. T.C. Arsene ${ }^{1}$, S. Pal ${ }^{2, a}$, P. J. Laz ${ }^{2}$, M. Taylor ${ }^{1 *}$

${ }^{1}$ Bioengineering Science Research Group,

School of Engineering Sciences,

University of Southampton,

Southampton,

SO17 1BJ

$\mathrm{UK}$

${ }^{2}$ Computational Biomechanics Lab,

Department of Engineering,

University of Denver,

2390 S. York,

Denver,

CO 80208

USA

*Corresponding Author:

Professor Mark Taylor

Bioengineering Science Research Group,

University of Southampton,

Highfield

Southampton

SO17 1BJ

UK

Tel: ++ 44 (0)2380 597660

Fax: ++ 44 (0)2380 593230

Email: mtaylor@soton.ac.uk

Word Count:

Abstract: 162

Introduction-Discussion: 3303

${ }^{\mathrm{a}}$ Currently, Department of Bioengineering, Stanford University, Stanford, CA 


\section{ABSTRACT}

55 Explicit finite element (FE) and multi-body dynamics (MBD) models have been developed to evaluate total 56 knee replacement (TKR) mechanics as a complement to experimental methods. In conjunction with these 57 models, probabilistic methods have been implemented to predict performance bounds and identify important 58 parameters, subject to uncertainty in component alignment and experimental conditions. Probabilistic 59 methods, like Advanced Mean Value (AMV) and Response Surface Method (RSM), provide an efficient 60 alternative to the gold standard Monte Carlo Simulation Technique (MCST). The objective of the current 61 study was to benchmark models from 3 platforms (2 FE and $1 \mathrm{MBD}$ ) using various probabilistic methods by 62 predicting the influence of alignment variability and experimental parameters on TKR mechanics in 63 simulated gait. Predicted kinematics envelopes were on average about $2.6 \mathrm{~mm}$ for anterior-posterior (AP) 64 translation, $2.9^{\circ}$ for internal-external (IE) rotation and $1.9 \mathrm{MPa}$ for peak contact pressure for the various 65 platforms and methods. Based on the good agreement with the MCST, the efficient probabilistic techniques 66 may prove useful in the fast evaluation of new implant designs, including considerations of uncertainty, e.g. 67 misalignment.

69 Keywords: TKR, Kinematics, Contact mechanics, Knee mechanics, Probabilistic methods, Simulation 


\section{Introduction}

Computational analysis has been used in orthopaedic studies since the 1980s (e.g. Huiskes and Chao, 1983; Prendergast, 1997). Modelling the behaviour of total knee replacements (TKR) is challenging, as the stresses generated within the prosthesis and the supporting bone are a function of the kinematics, and the kinematics in turn are a function of the implant design, the relative position of the components and the balance of the soft tissues. Early studies used quasi-static loading conditions, effectively ignoring the et al., 2001) and explicit (Godest et al 2002, Halloran et al., 2005a; Halloran et al., 2005b) FE or rigid body (Fregly et al., 2003) modelling techniques to simultaneously predict kinematics and stresses.

Clinical and experimental simulator studies have reported substantial variability in TKR kinematics

(DesJardins et al., 2000; Mahaluxmivala et al., 2001; Dennis et al., 2003; Zihlmann et al., 2005).

Computational models have shown good agreement with experimental simulator results (Godest et al., 2002,

Halloran et al., 2005a; 2005b), providing important model validation as well as additional insight into

performance metrics that are difficult to measure experimentally (like contact pressure). While models are typically developed for 'average' conditions, they are an ideal platform to explore the influence of variability, as a consequence of either patient-, surgery- or component-related parameters. Parametric studies

al., 2001). Probabilistic evaluations assess multiple parameters simultaneously, and represent each input parameter as a distribution in order to predict an envelope of performance. In addition to accounting for orthopaedic components (Browne et al., 1999; Nicolella et al., 2001; Dar et al., 2002). More recently, the application of efficient probabilistic techniques has been used to assess the performance of TKR (Laz et al. 2006a; 2006b). The advanced mean value (AMV) method (corroborated with a 1000 trial Monte Carlo Simulation Technique (MCST)) assessed the impact of experimental variability in a knee wear simulator on 
predicted TKR mechanics by determining the performance envelopes of joint kinematics and contact

98 parameters. In the Laz et al. (2006a) study, 8 component alignment parameters and 4 experimental 99 parameters were represented as Normal (Gaussian) distributions and used with probabilistic methods to 100 assess the response of the TKR model

A variety of software packages exist for FE, multi-body dynamics (MBD) and probabilistic methods. This study seeks to evaluate whether model predictions would differ depending on the software platform used, to evaluate the robustness of the computational and probabilistic approach. The aims of this benchmark study are: to perform probabilistic TKR mechanics predictions using three different platforms combining FE or MBD solvers with statistical/probabilistic analysis, and so to evaluate the accuracy and efficiency of various platforms and probabilistic methods.

\section{Methods}

109 This study will evaluate performance envelopes for tibial anterior-posterior (AP) translation, tibial internal-

110 external (IE) rotation and tibial peak contact pressure during a standard gait cycle using explicit FE and 111 rigid body modelling techniques, and will compare results of the AMV and response surface methods 112 (RSM) to those from MCST.

\section{$113 \quad 2.1$ Deterministic modelling of the in-vitro simulator}

114 In all three analyses, an isolated tibiofemoral joint was modelled (Figure 1). The loading conditions 115 represented the force-controlled gait simulation of a knee wear simulator (Walker et al., 1997; DesJardins et al., 2000; ISO Standard 14243-1, 2000).

Models were developed from CAD parts of a semi-constrained, fixed-bearing, cruciate-retaining TKR. The distal surface of the tibial insert was supported in the inferior-superior (IS) direction, whilst loading conditions applied to the insert included an anterior-posterior (AP) load and internal-external (IE) torque. The axial load was applied along the inferior superior axis and the flexion angle was applied along the femoral axis. Varus-valgus (VV) and tilt of the insert were both constrained, and AP, medial-lateral (ML) and IE degrees of freedom (DOF) were unconstrained. The femoral component was constrained in IE, ML 
and AP DOF, unconstrained in VV and IS DOF, and displacement-controlled flexion rotation was applied.

124 There is an axial compressive force and a flexion angle applied. The model included simulated soft-tissue 125 constraint present in the knee simulator consisting of four springs constraining the insert AP displacement 126 and IE rotation (Figure 1).

Three different analysis packages were investigated in order to build a baseline deterministic model, both to benchmark the results and to provide a platform for further stochastic modelling.

\section{i) Abaqus/Explicit}

An explicit FE model of the TKR (Laz et al., 2006a) was developed in Abaqus/Explicit (Simulia, Providence, RI). The tibial insert was represented with three-dimensional, 8-nodal hexahedral elements $(\sim 8500)$, and rigid triangular surface elements $(\sim 19,000)$ were used for the femoral component. A convergence study was implemented before the probabilistic analysis to confirm the mesh density was acceptable (Halloran et al., 2005a). The femoral and tibial components were represented as rigid bodies, with a nonlinear pressure-overclosure relationship (Halloran et al., 2005b).

\section{ii) MSC ADAMS}

The MBD model was developed in ADAMS (MSC Software Corporation, Santa Ana, CA). The deterministic study principally involved modelling with the ADAMS/View module. In order to model contact, ADAMS uses an 'IMPACT' function, which relates normal reaction force to interpenetration displacement and can be used for extremely high-speed single-contact 'surrogate' models (e.g. Lin et al., 2009). However, if contact-pressure information is required, then a unified single-body contact force is not adequate (as it does not include information about the force distribution), so instead the articulation must be 'discretised' into multiple contacts across the surface to estimate the local contact force contribution at each location. Various established penalty-based algorithms exist for such a distributed contact model, for example, the elastic foundation model (Fregly et al., 2003).

iii) PAM-CRASH 
An explicit FE model (Godest et al, 2002) was developed in PAM-CRASH (ESI/PAM System

International, France). Both the femoral component and the tibial polyethylene insert were modelled as

rigid bodies using four-nodded shell elements. An advanced penalty-method based contact algorithm (contact element 44 in PAM-CRASH) was used to model the contact between the two components.

This algorithm operates by penalising the geometric penetration of the slave nodes by counteracting forces proportional to the penetration depth of the tibia and a user-specified penalty factor.

\subsection{Probabilistic modelling}

Probabilistic modelling techniques are utilized to account for uncertainty in multiple input parameters and to predict a distribution of performance. In the present probabilistic evaluations, twelve experimental parameters (Table 1) representing component alignment, loading, and experimental conditions were evaluated. The experimental parameters included four translations and four rotations of the femoral component and tibial insert. The rotations and translations defined the position of the femoral component and tibial insert relative to the fixed rotational axes (Figure 1). In addition, experimental set-up parameters (the spring stiffness constant, the ML separation of the springs, and ML load split) and friction were also included. The levels of variability (Table 1) were estimated for a knee simulator with standard deviations of $0.5 \mathrm{~mm}$ for translational and $1^{\circ}$ for rotational alignment, respectively (Laz et al., 2006a). Each of the parameters was assumed to be independent and normally-distributed.

Analyses were performed using three probabilistic methods (MCST, RSM and AMV) in three probabilistic platforms:

i) Nessus/Abaqus: The Nessus probabilistic software (SwRI, San Antonio, TX) was integrated with the FE model through custom scripting. The AMV and MCST methods were applied.

ii) Insight/ADAMS: The statistical analysis module ADAMS/Insight was used with the MBD solver. RSM and MCST methods were applied.

iii) PAM-OPT/PAM-CRASH: The optimization software PAM-OPT was used in conjunction with the PAM-CRASH model. A 1000-trial MCST analysis was performed. Following this, a first order RSM 
implemented in MATLAB (Mathworks, Inc., Natick, MA) was implemented with a reduced set of MCST points (25, 50 and 100).

A brief description of the probabilistic methods utilized is included to highlight the differences in accuracy, efficiency and robustness. MCST involves repeated sampling of the input parameters according to their distributions, with the accuracy of the resulting output distribution dependent on the number of trials performed. The MCST is a robust method which provides accurate results with many trials, but is computationally expensive.

For RSM (Box and Wilson, 1951), an analytic function of the input variables is fitted to approximate the output parameter over the sample space based on an initial set of model evaluations. The initial set of model evaluations can be performed at random, but better results are achieved by distributing the trials regularly across the sample space. In the most basic case, a low order polynomial and regression techniques may be used to determine the term coefficients. Subsequently, MCST is performed using this response surface equation, instead of performing additional evaluations (resulting in lower computational cost). RSM works best when the output response is well-represented by the analytic function, i.e. relatively linear, smooth and monotonic models; highly non-linear functions are not well-represented. The higher the order of the response surface equation used, the more terms are needed; hence more samples are needed to achieve a good fit with the regression. Beyond second-order terms, this becomes impractical for many models. In the current study, the TKR model is expected to be relatively linear within the small perturbation range being studied here; accordingly, a first order (linear) response surface equation was evaluated based on initial sample sizes of 25, 50 and 100 trials and subsequent MCST of 1000 evaluations of the response surface equation.

The AMV method is an optimization-based method utilizing a mean value approximation augmented with higher-order terms to determine the response at a specified probability level (Wu, et al., 1990). Although an approximate technique, the AMV method has been shown to work well for well-behaved monotonic systems (Easley, 2007) and has been implemented in prediction of performance envelopes for TKR (Laz et al., 2006a)). The Mean Value (MV) family of methods begin with a local first order (linear) 
approximation of the function about the mean value of the input function - whereas RSM builds a global model. The MV model is suitable for fairly linear problems, but is not accurate for non-linear behaviour; its main practical use is as the basis for the subsequent AMV. The AMV method takes the linear model derived by the MV method, and attempts to include corrective terms to approximate the higher-order effects. More exactly, it takes the MV prediction and using data from the calculated Most Probable Point (MPP) of interest, corrects this value for a single level of desired probability (or desired output) which gives the corrected AMV estimate of the output. The calculated MPP is the global maximum of the probability distribution function of all the possible different points of failure along a 'limit state' (i.e. failure) function. The AMV essentially gives a more accurate representation at one localised point of the possibility space, whereas RSM gives a less accurate representation, but is valid across the entire possibility space.

For each platform, the results of the combined probabilistic/mechanical modelling were a bounded response representing the $1^{\text {st }}$ and $99^{\text {th }}$ percentiles over the gait cycle for the performance metrics: AP translation, IE rotation and peak CP. AP translation and IE rotation are reported relative to the 'settled' reference positions of the components. Additionally, sensitivity factors, representing the effect of varying each input parameter on the output response, identify the most and least important parameters. The sensitivities at each time point were calculated as the linear regression-fit of the input variables ('normalised' based on standard deviations) to the MCST results. The absolute magnitude of the sensitivity values at each time point were then averaged over the gait cycle to give a mean absolute value. To provide an overall indication of importance, the reported sensitivity factors are the normalised, averaged absolute values over the gait cycle.

\section{Results}

\subsection{Comparison of the deterministic results}

The deterministic results for AP translation, IE rotation and peak $\mathrm{CP}$ showed good agreement between the Abaqus, ADAMS and PAM-CRASH platforms and with the experimental results (Figure 2). To quantify the differences between the different model results and the experimental data, the Root Mean 
Square (RMS) variations over the gait cycle were computed between each model and the experimental data and between models (Table 2). RMS differences between model and experiment averaged $0.6 \mathrm{~mm}$ and $1.0^{\circ}$ for AP translation and IE rotation, respectively. RMS differences between models were of a similar magnitude. While the general behaviour in predicted peak CP pressure was similar for all models, there were differences in the predicted magnitude, mainly due to the difference in contact formulations between platforms.

Computation time for an analysis was approximately 3 minutes in Abaqus/Explicit (Intel Pentium 4 3GHz, 2Gb RAM), 4 minutes in PAM-CRASH (Intel Pentium 4 3.20GHz, 2Gb RAM) and 6 minutes in ADAMS (Intel Pentium $43 \mathrm{GHz}, 2 \mathrm{~Gb} \mathrm{RAM}$ ). Notably, for all three platforms, the analysis time is sufficiently low to allow larger-scale probabilistic studies.

\subsection{Comparison of the probabilistic results}

Envelopes of the $1 \%$ to $99 \%$ bounds were predicted for the TKR performance measures using the three platforms: Nessus/Abaqus, Insight/ADAMS, PAM-OPT/PAM-CRASH. The MCST comparisons (Figure 3) showed good agreement in trend and performance envelope size for the various platforms. Similar behaviour was observed for the response surface and AMV methods (Figures 4 and 5).

The differences between the methods (MCST versus AMV and MCST versus RSM) for the average and the maximum bounds for the various platforms (Table 3) were within 5\% for kinematics. On average, envelope sizes were within approximately $2.6 \mathrm{~mm}$ for AP translation and $2.9^{\circ}$ for IE rotation. For all platforms, the kinematic envelopes were larger during stance than swing phase. While differences in the magnitudes of peak CP were present from the deterministic models, the MCST probabilistic bounds were similarly shaped, with differences between the smallest average and the largest average peak contact pressure of 1.3 MPa: 1.4 MPa for Nessus/Abaqus, 2.5 MPa for ADAMS/Insight and 2.8 MPa for PAMOPT/PAM-CRASH.

The RSM was evaluated to characterize the sensitivity of the response surface equation to the number of initial trials, specifically 25, 50 and 100 trials using PAM-OPT/PAM-CRASH and Insight/ADAMS platforms (Figure 4). The kinematic (AP and IE) results for PAM-OPT/PAM-CRASH 
showed agreement between all RSM models and the MCST results, implying that an RSM based on 25 trials was sufficient. However, the RSM predictions of peak CP were more sensitive to the number of trials used. While good agreement could be achieved with PAM-OPT/PAM-CRASH for the maximum value of peak CP over the gait cycle using the 100 trial RSM, the secondary peak at $10 \%$ gait, and the lower swing phase pressures were not well captured (Figure 4c). For the Insight/ADAMS platform, similar RSM results were observed where 25 trials were sufficient to model kinematics (Table 3), but 100 trials were not sufficient to characterize the swing phase (Figure 4d). Similar results were observed for the AMV method (Figure 5, Table 3), where maximum differences in the size of the predicted AP and IE ranges were $0.07 \mathrm{~mm}$ and $0.18^{\circ}$ for the AMV and MCST methods. As contact pressure during swing phase exhibits more non-linear behaviour, approximate methods like RSM and AMV were less accurate (Figures 4 and 5). Regarding computation time, MCST (1000 trials) ranged from 50-100 hours, AMV results for a single performance measure (173 trials) required $\sim 9$ hours, RSM (25 trials) required 2 hours and RSM-100 required 8 hours.

The sensitivities, reported as average of the absolute values over the gait cycle (Figure 6), illustrated the relative impact of the parameters on AP translation, IE rotation and peak CP. For AP translation and IE rotation, good agreement in important parameters and their magnitudes was obtained with the three software platforms. Insert tilt and femoral IE alignment were the most important parameters affecting AP translation and IE rotation respectively. The sensitivity factors for peak CP identified four contributing parameter with relatively equal contributions: initial femoral flexion-extension, femoral IE, insert tilt and VV alignment.

\section{Discussion}

In this benchmarking study, predictions of TKR performance under simulated gait conditions were performed with three FE/MBD platforms using different probabilistic methods. By comparing the results from the various platforms, this study evaluated whether the choice of computational platform affected the predicted results, as well as the accuracy and efficiency of the MCST, RSM and AMV probabilistic methods.

Notably, the deterministic results for AP translation, IE rotation and peak $\mathrm{CP}$ obtained using FE and MBD software platforms showed similar patterns throughout the gait cycle and were in close agreement 
with experimental kinematic data and between platforms (Figure 2). The RMS differences between the models were on average of $0.5 \mathrm{~mm}$ in AP translation and $0.9^{\circ}$ in IE rotation. Greater differences were observed in peak CP than in the kinematic measures, with RMS difference being up to $2.1 \mathrm{MPa}$ between PAM-CRASH and Abaqus. The highest difference was between ADAMS and PAM-CRASH of 3.0 MPa. Differences in the magnitude of $\mathrm{CP}$ were attributed to the different software platforms and the methods of implementing contact in a rigid body simulation. Factors which are complicit in these differences include the contact model parameters, the frictional parameters, and the effects of inertia within the software platforms. Nonetheless, in spite of these small differences, overall the trends and magnitudes of the responses matched favourably.

Supported by the accuracy of the deterministic results and fast computational times, probabilistic analyses were performed using thee platforms: Nessus/Abaqus, Insight/ADAMS and PAM-OPT/PAMCRASH. MCST was carried out for all models and predicted similarly sized performance envelopes were obtained for the kinematics in all three methods. Larger differences were observed in the $1 \%$ and $99 \%$ envelopes for peak $\mathrm{CP}$, but these are again attributed to deterministic model differences than probabilistic methods. MCST computational times were similar for the three different platforms.

The increased computational times associated with probabilistic FE/MBD modelling represent an important barrier to incorporating such techniques in the design-phase evaluation of TKR implants. For example, the 1000-trial MCST implemented in PAM-CRASH/PAM-OPT required 4 days of computational time. This highlights the need to implement and validate more efficient alternatives to the 'gold standard' of MCST. Comparison of RSM envelope sizes to MCST yielded an average difference of $0.08 \mathrm{~mm}(2.9 \%)$ and $0.03^{\circ}(1.0 \%)$ in AP translation and IE rotation, respectively (Figures $4 \mathrm{a}$ and $4 \mathrm{~b}$, Table 3 ) and computational costs substantially reduced to 4 hours for the 50-trial RSM analysis. Similarly, comparison of AMV envelopes to MCST in Nessus/Abaqus yielded an average difference of $0.08 \mathrm{~mm}(3.7 \%)$ and $0.1^{\circ}(3.6 \%)$ in AP translation and IE rotation, respectively (Figure 5, Table 3). The computational time required for the AMV analyses were $\sim 9$ hours. It is important to note that the RSM and AMV methods are less robust for highly non-linear or non-monotonic systems. This explains the greater differences in CP results during the 
swing phase (Figures $4 \mathrm{c}, 4 \mathrm{~d}$ and $5 \mathrm{c}$ ), when minimal constraint (due to small joint loads) resulted in a more non-linear system.

The efficient RSM and AMV methods provide distinct advantages compared to one another. The AMV method provides local approximation of system behaviour at pre-defined points in the possibility space, while RSM presents a global approximation across the entire possibility space. The RSM is less accurate than AMV at targeted local design point(s), but is more flexible than AMV in predicting behaviour across the entire possibility space. An RSM analysis requires a fixed number of trials regardless of the number of desired output measures; in contrast, an AMV analysis requires an additional trial for every desired output measure (e.g. AP translation, IE rotation, or CP) and probability level (e.g. 1\% or 99\%).

Independent of platform, the sensitivity factors identified the same set of important input parameters (Figure 6). Specifically, insert tilt was the greatest contributor to AP translation, while femoral IE alignment had the largest sensitivity factor for IE rotation. In addition to insert tilt and femoral IE alignment, two other alignment parameters were important to contact pressure. These factors have been identified in clinical studies [Catani et al., 2006; Anouchi et al., 1993] and underscore the importance of component alignment to TKR mechanics.

In closing, this study performed benchmark comparisons of FE and MBD, as well as probabilistic software packages and generally found good agreement in results independent of the methods. Our results suggest that researchers can use both FE and MBD-based approaches and probabilistic methods besides MCST, with confidence that the results will be comparable across different platforms. The accuracy of the efficient probabilistic methods, e.g. RSM and AMV, can aid in the quicker design phase evaluation of the robustness of TKR implants to surgical and environmental variables.

\section{References}

Anouchi YS, Whiteside LA, Kaiser AD, Milliano MT. "The effects of axial rotational alignment of the femoral component on knee stability and patellar tracking in total knee arthroplasty demonstrated on autopsy specimens". Clinical Orthopaedics and Related Research. 1993;287:170-177.

Besier TF, Gold GE, Delp SL. "The influence of femoral internal and external rotation on cartilage stresses within the patellofemoral joint”. Journal of Orthopaedic Research. 2008; Vol. 26:1627-1635. 
Box GEP, Wilson KB. "On the experimental attainment of optimum conditions". Journal Royal Statistical Society Series B, 1951. 13, 1-45.

Browne, M., Langley, R.S., Gregson, P.J.. "Reliability theory for load bearing biomedical implants". Biomaterials. 1999. Vol. 20, 1285-1292.

Catani F, Fantozzi S, Ensini A, Leardini A, Moschella D. and Gianninni S. "Influence of tibial component posterior slope on in vivo knee kinematics in fixed-bearing total knee arthroplasty". Journal of Orthopaedic Research. 2006; Vol. 24: 581-587.

Dar, F.H., Meakin, J.R., Aspden, R.M., 2002. "Statistical methods in finite element analysis." Journal of Biomechanics 35(9), 1155-1161.

Dennis, D.A., Komistek R.D., Mahfouz, M.R. 2003. "In vivo fluoroscopic analysis of fixed-bearing total knee replacements". Clinical Orthopaedics and Related Research. 410, 114-130.

DesJardins, J.D., Walker, P.S., Haider, H., Perry, J., 2000. "The use of a force-controlled dynamic knee simulator to quantify the mechanical performance of total knee replacement designs during functional activity". Journal of Biomechanics 33, 1231-1242.

Dorr, L.D. and R.A. Boiardo, 1986. "Technical considerations in total knee arthroplasty". Clinical Orthopaedics and Related Research 205, 5-11.

Elias, J.J., Rai, S.P., Ciccone II W.J., 2008. "In vitro comparison of tension and stiffness between hamstring tendon and patella tendon grafts". Journal of Orthopaedic Research 26(11), 1506-1511.

Fregly, B.J., Bei, Y., Sylvester, M.E., 2003. "Experimental evaluation of an elastic foundation model to predict contact pressures in knee replacements", Journal of Biomechanics 36, 1659-1668.

Easley, S.K., Pal, S., Tomaszewski, P.R., Petrella, A.J., Rullkoetter, P.J., Laz, P.J., 2007. Finite elementbased probabilistic analysis tool for orthopaedic applications. Computer Methods and Programs in Biomedicine 85, 32-40.

Fregly, B.J., Sawyer, W.G., Harman, M.K and S.A. Banks, 2005. "Computational wear prediction of a total knee replacement from in vivo kinematics", Journal of Biomechanics 38, 305-314.

Godest, A.C., Beaugonin, M., Haug, E., Taylor, M., Gregson, P.J., 2002. "Simulation of a knee joint replacement during gait cycle using explicit finite element analysis." Journal of Biomechanics 35, 267-275.

Halloran, J.P., Petrella, A.J., Rullkoetter, P.J., 2005a. "Explicit finite element modelling of total knee replacement mechanics." Journal of Biomechanics 38, 323-331.

Halloran, J.P., Easley, S.K., Petrella, A.J., Rullkoetter, P.J., 2005b. "Comparison of deformable and elastic foundation finite element simulations for predicting knee replacement mechanics." Journal of Biomechanical Engineering 127, 813-818.

Huiskes, R. and Chao, E. Y. S., 1983, A survey of finite element analysis in orthopaedics biomechanics: the first decade, Journal of Biomechanics 16, 385-409.

Li, G., Lopez, O., Rubash, H., 2001. "Variability of a three-dimensional finite element model constructed using magnetic resonance images of a knee for joint contact stress analysis“. Journal of Biomechanical Engineering 123, 341-346.

Isukapalli, S.S., Balakrishnan, S., and Georgopoulos. P., 2004. "Computationally efficient uncertainty propagation and reduction using the stochastic response surface method." 43rd IEEE Conference on Decision and Control, Atlantis, Bahamas.

ISO Standard 14243-2, 2000. "Wear of total knee-joint prostheses, part 2: methods of measurements." International Standards Organization.

McEwen, H.M.J., Fisher, J., Goldsmith, A.A., Auger, D.D., Hardaker C. and Stone, M.H., 2001. "Wear of fixed bearing and rotating platform mobile bearing knees subjected to high levels of internal and external tibial rotation“. Journal of Materials Science: Materials in Medicine 12(10-12), 1049-1052.

Otto, K., J.J. Callaghan and T.D. Brown, 2001. "Mobility and contact mechanics of a rotating platform total knee replacement". Clinical Orthopaedics and Related Research 392, 24-37.

Prendergast, P.J., 1997, Finite element models in tissue mechanics and orthopedic implant design, Clinical Biomechanics 12 (6), 343-366.

Kawanabe, K., Clarke, I.C., Tamura, J., Akagi, M., Good, V.D., Williams, P.A., Yamamoto, K., 2001. "Effects of A-P translation and rotation on the wear of UHMWPE in a total knee joint simulator." Journal of Biomedical Materials Research 54, 400-406. 
Kurth, R.E., Woods, K.S., 1992. "Probabilistic damage tolerant analysis for fatigue critical aircraft components." ASME, Aerospace Division 28, 89-97.

Laz, P.J., Pal, S., Halloran, J.P., Petrella, A.J., Rullkoetter, P.J., 2006a. "Probabilistic finite element prediction of knee wear simulator mechanics." Journal of Biomechanics 39, 2303-2310.

Laz, P.J., Pal S., Fields, A., Petrella, A.J. and Rullkoetter, P. J., 2006b, "Effects of knee simulator loading and alignment variability on predicted implant mechanics: a probabilistic study", Journal of Orthopaedic Research 24, 2212-2221.

Lin, Y.C., Haftka R.T., Queipo, N.V., Fregly, B.J., 2009. "Two-Dimensional Surrogate Contact Modeling for Computationally Efficient Dynamic Simulation of Total Knee Replacements." Journal of Biomechanical Engineering 131, 041010-1.

Mahaluxmivala, J, Bankes, M.J., Nicolai, P., Aldam, C.H., Allen, P.W., 2001. "The effect of surgeon experience on component positioning in 673 Press Fit Condylar posterior cruciate-sacrificing total knee arthroplasties." Journal of Arthroplasty 16 (5), 635-675.

McEwen, H.M., Barnett, P.I., Bell, C.J., Farrar, R., Auger, D.D., Stone, M.H., Fisher, J., 2001. "The influence of design, materials and kinematics on the in vitro wear of total knee replacements." Journal of Biomechanics 38, 357-365.

Melis, M.E., Zaretsky, E.V., August, R., 1999. "Probabilistic analysis of aircraft gas turbine disk life and reliability." Journal of Propulsion and Power 15(5), 658-666.

Nicolella, D.P., Thacker, B.H., Katoozian, H., Davy, D.T., 2001. "Probabilistic risk analysis of a cemented hip implant." ASME Bioengineering Division 50, 427-428.

Taylor, M. and Barrett, D.S., 2003. "Explicit finite element simulation of eccentric loading in total knee replacement ." Clinical Orthopaedic Related Research 414, 162-171.

Walker, P.S. and A. Garg, 1991. "Range of motion in total knee arthroplasty". Clinical Orthopaedics and Related Research 262, 227-235.

Walker, P.S., Blunn, G.W., Broome, D.R., Perry, J., Watkin, S.A., Sathasivam, S., Dewar, M.E., Paul, J.P., 1997. "A knee simulating machine for performance evaluation of total knee replacements." Journal of Biomechanics 30, 83-89.

Wu, Y.T., Millwater, H.R., Cruse, T.A., 1990. "Advanced Probabilistic Structural-Analysis Method for Implicit Performance Functions." AIAA Journal 28, 1663-1669.

Zhang, Y., Liu, Q., 2002. Reliability-based design of automobile components. Proceedings of the Institute of Mechanical Engineers, Part D. Journal of Automobile Engineering 216(6), 455-471.

Zihlmann M.S., Stacoff A., Romero, I., Kramers-de Quervain, Stussi E., 2005. "Biomechanical background and clinical observations of rotational malalignment in TKA-literature review and consequences" Clinical Biomechanics. Vol. 20: 661-668. 
Figure 1. Probabilistic study parameters in the FE model of TKR. (For the definition of the abbreviations used, see Table 1).

430

431

432

433

434

435

436

437

438

439

440

441

442

443

444

445

446

447

448

449

450

451

452

453

454

455

456

457

458

459

460

461

462

463

464

465

466

467

Figure 2. Tibial kinematics and contact pressure from the three models: Abaqus/Explicit, ADAMS, and PAM-CRASH. a) AP translation (+anterior/-posterior), b) IE rotation (+external/-internal), and c) peak contact pressure.

Figure 3. Comparison of predicted envelopes (1-99\%) from MCST implemented in Nessus/Abaqus, ADAMS/Insight, and PAM-OPT/PAM-CRASH. a) AP translation (+anterior/-posterior), b) IE rotation (+external/-internal), and c) peak contact pressure.

Figure 4. Comparison of predicted envelopes (1-99\%) for RSM based on 25, 50, and 100 trials and MCST (solid line) with 1000 trials. Results from PAM-OPT/PAM-CRASH for a) AP translation (+anterior/posterior), b) IE rotation (+external/-internal), and c) peak contact pressure and from ADAMS/Insight for d) peak contact pressure.

Figure 5. Comparison of predicted envelopes (1-99\%) for AMV and MCST with 1000 trials using the Nessus/Abaqus platform. a) AP translation (+anterior/-posterior), b) IE rotation (+external/-internal), and c) peak contact pressure

Figure 6. Sensitivity results for the various platforms represented by the averaged absolute values over the gait cycle for: a) AP translation (+anterior/-posterior), b) IE rotation (+external/-internal), and c) peak contact pressure.

Table 1. Input factors for probabilistic study from (Laz et al., 2006a).

Table 2. Root mean square (RMS) differences between the various platforms.

Table 3. Comparison of average and maximum ranges of predicted 1 and 99 percentile bounds for the various performance metrics and platforms. 


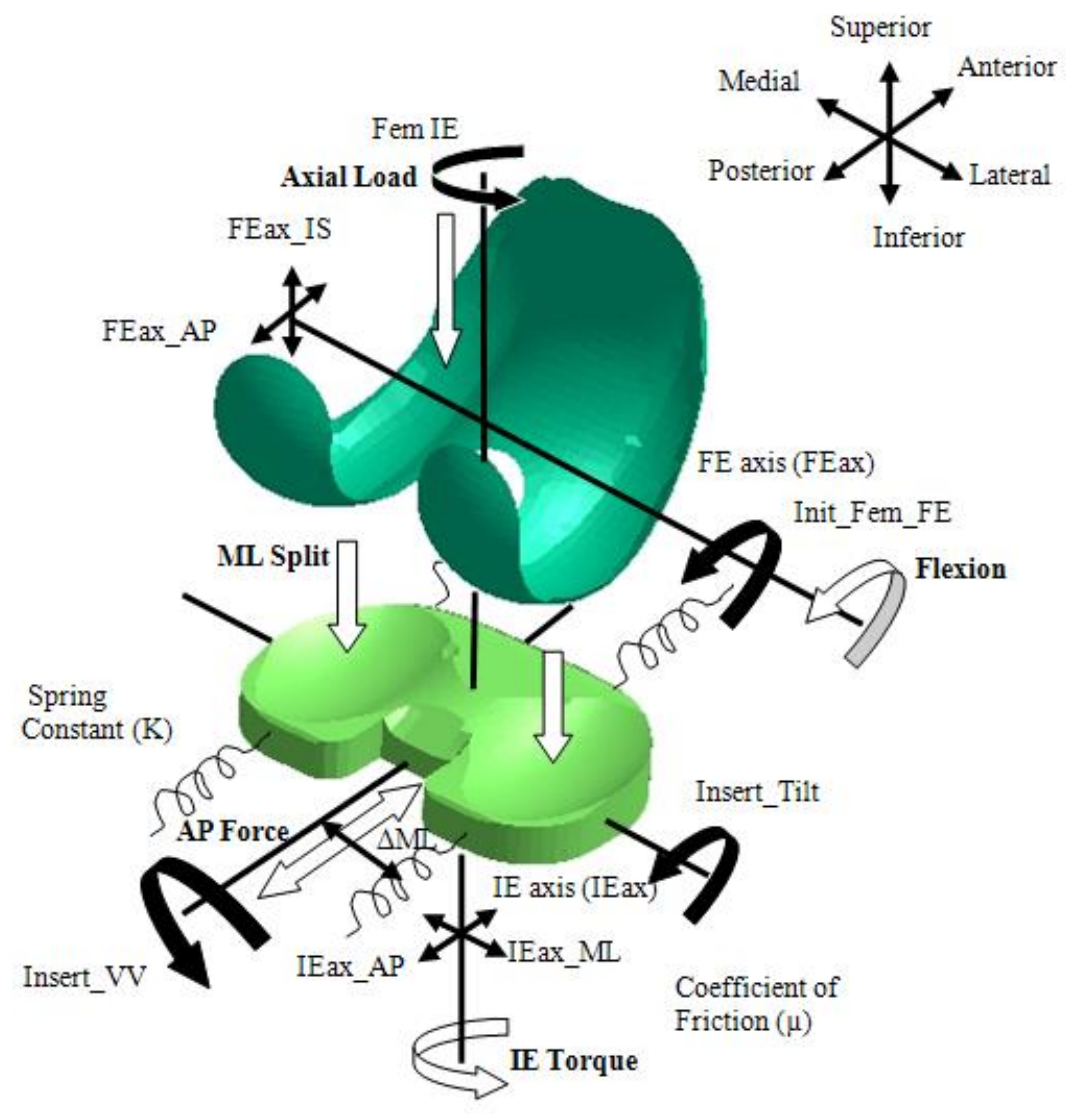

Figure 1. Probabilistic study parameters and the applied forces in the FE model of a TKR. (For the definition of the abbreviations used, see Table 1). 


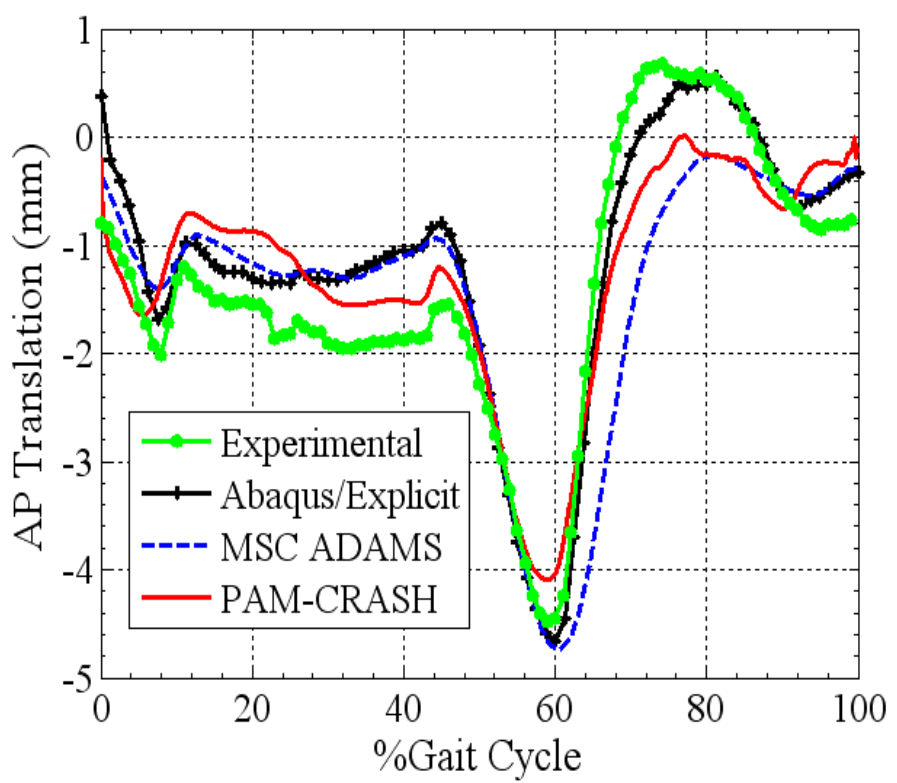

(a)

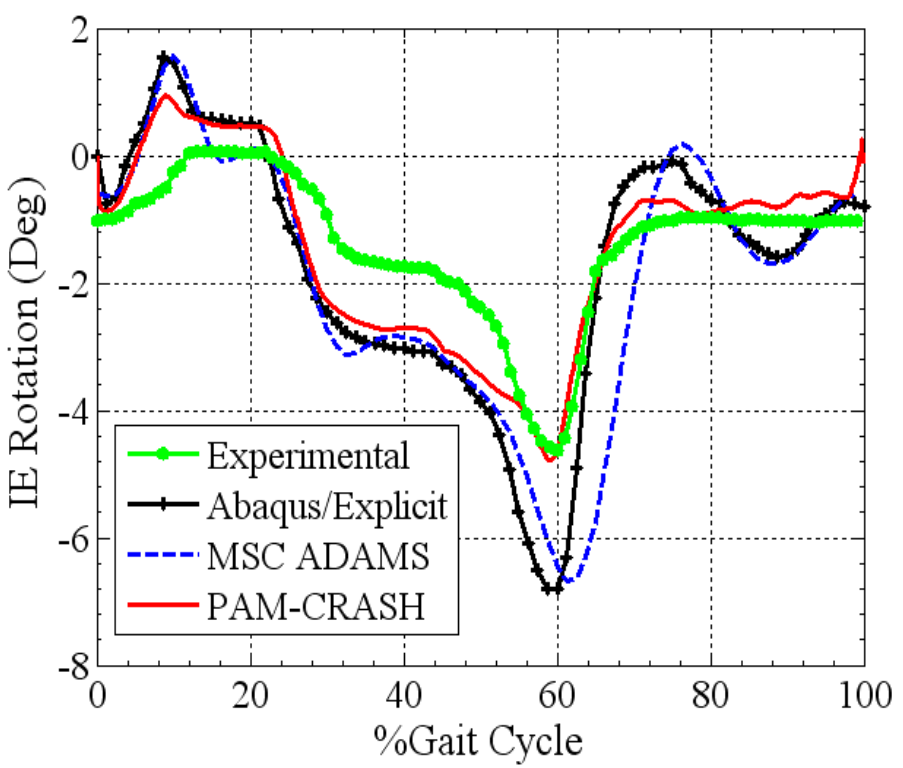

(b)

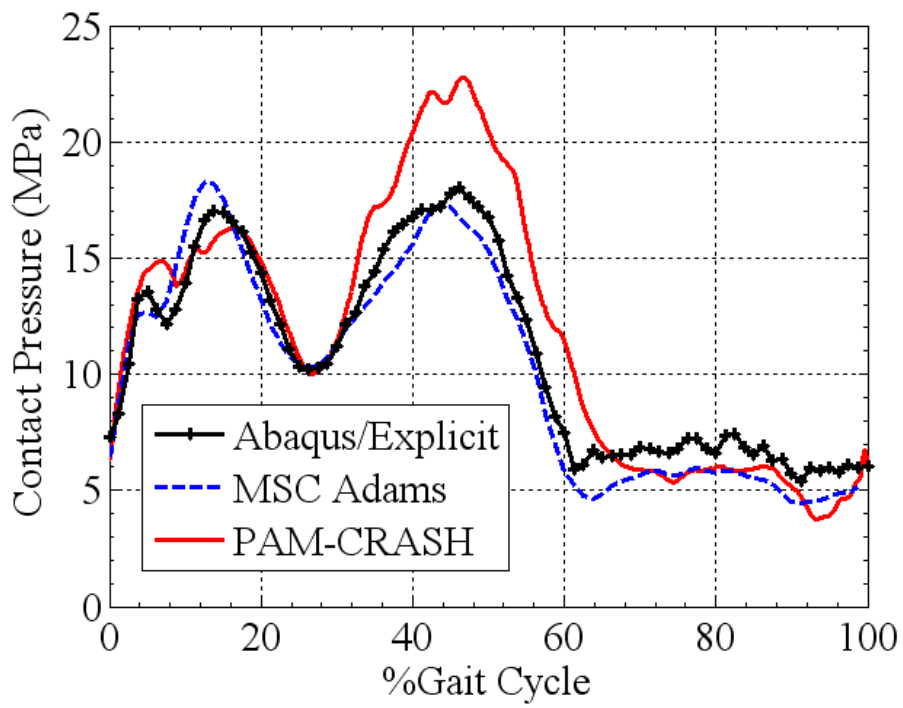

(c)

Figure 2. Kinematics and contact pressure from the three models: Abaqus/Explicit, ADAMS, and PAMCRASH. a) AP translation (+anterior/-posterior), b) IE rotation (+external/-internal), and c) peak contact pressure. 


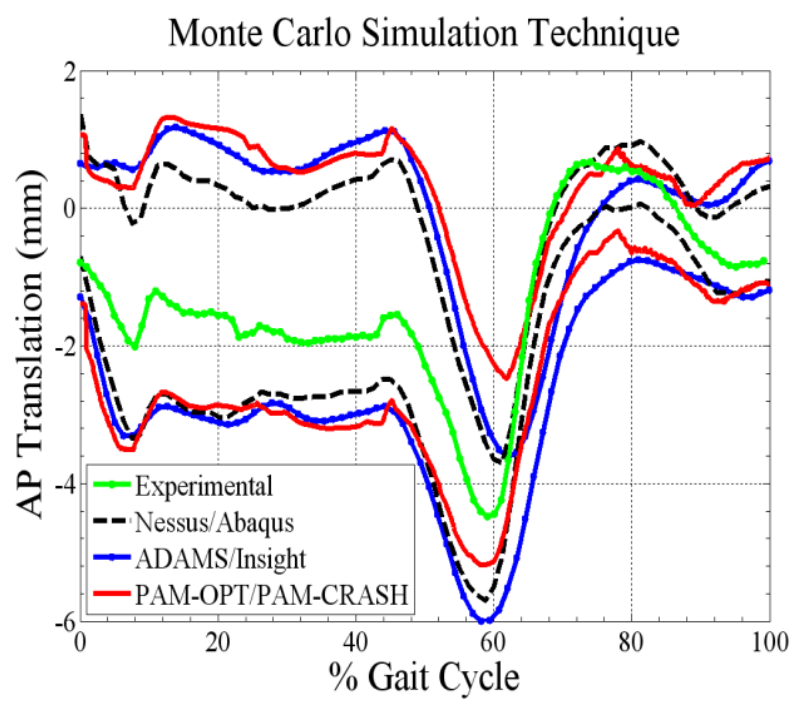

(a)

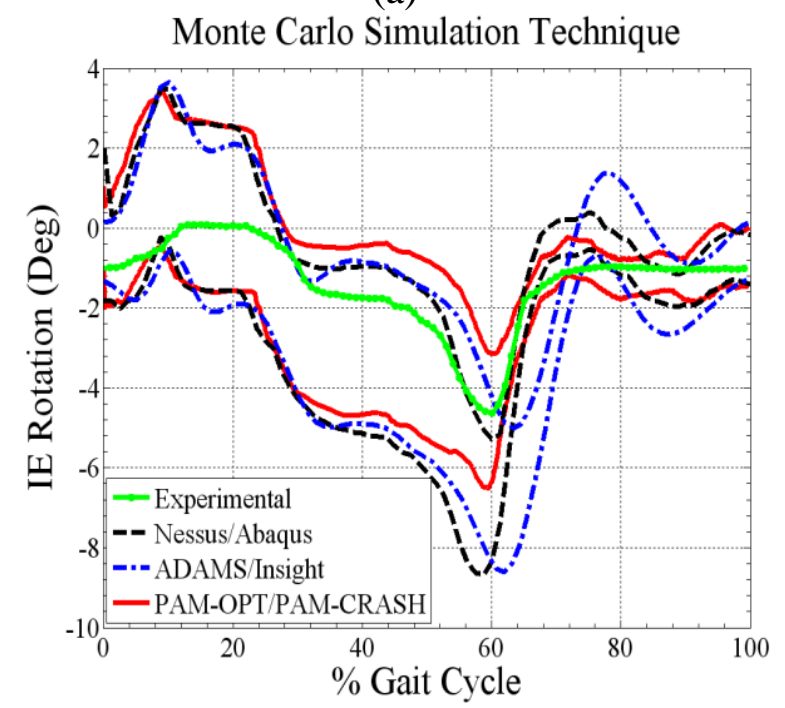

(b)

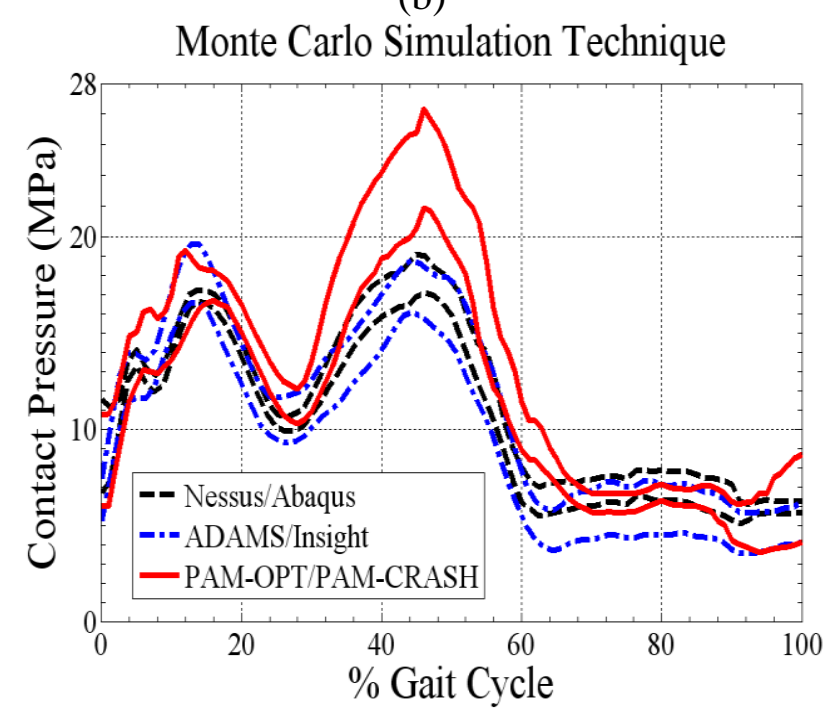

Figure 3. Comparison of predicted envelopes (1-99\%) from MCST implemented in Nessus/Abaqus, ADAMS/Insight, and PAM-OPT/PAM-CRASH. a) AP translation (+anterior/-posterior), b) IE rotation (+external/-internal), and c) peak contact pressure. 


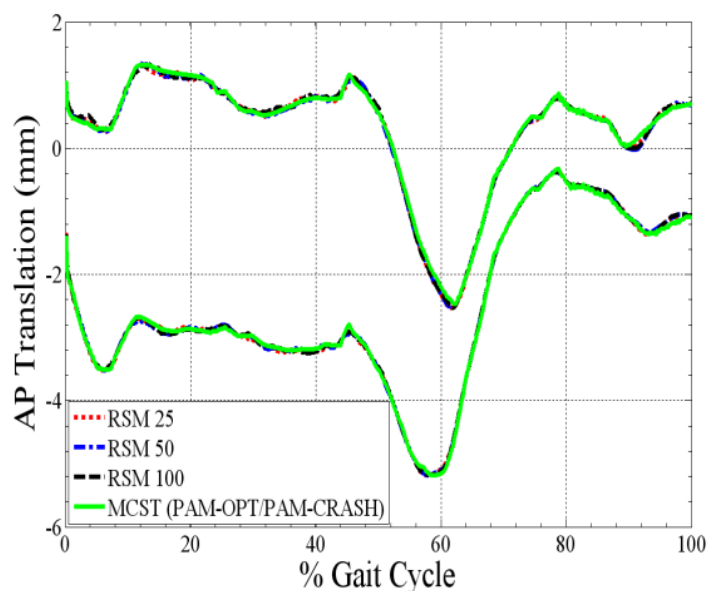

(a)

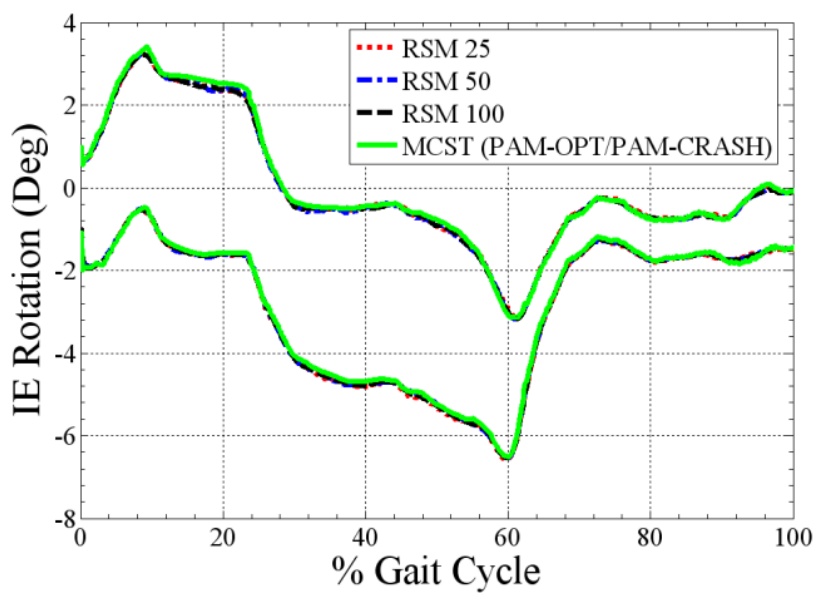

(b)

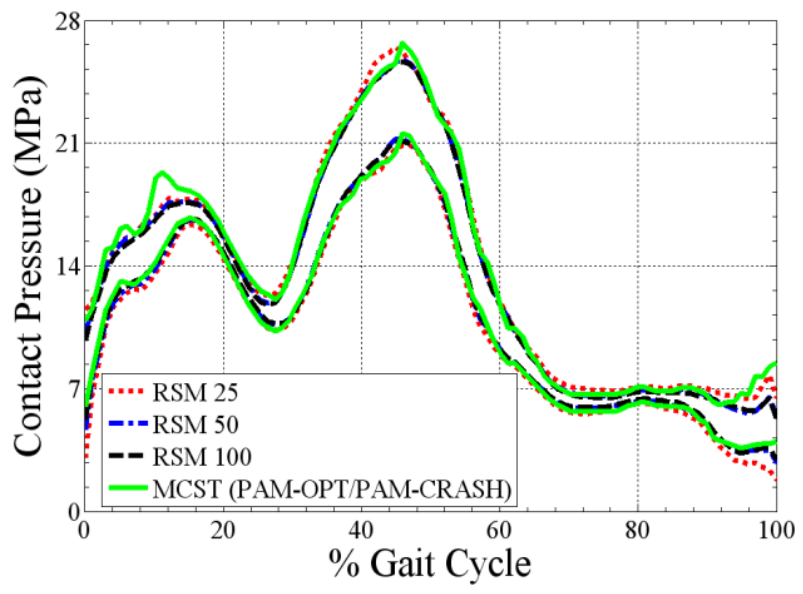

(c) 


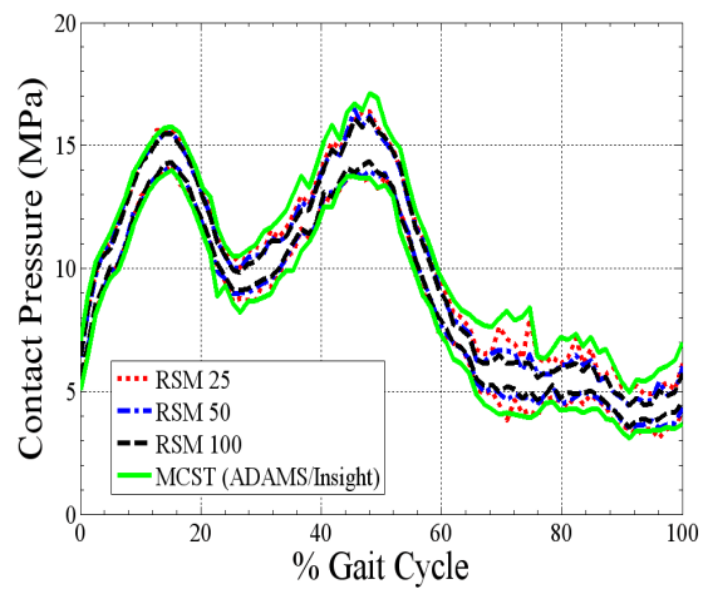

(d)

Figure 4. Comparison of predicted envelopes (1-99\%) for RSM based on 25, 50, and 100 trials and MCST (solid line) with 1000 trials. Results from PAM-OPT/PAM-CRASH for a) AP translation (+anterior/posterior), b) IE rotation (+external/-internal), and c) peak contact pressure and from Insight/Adams for d) peak contact pressure. 

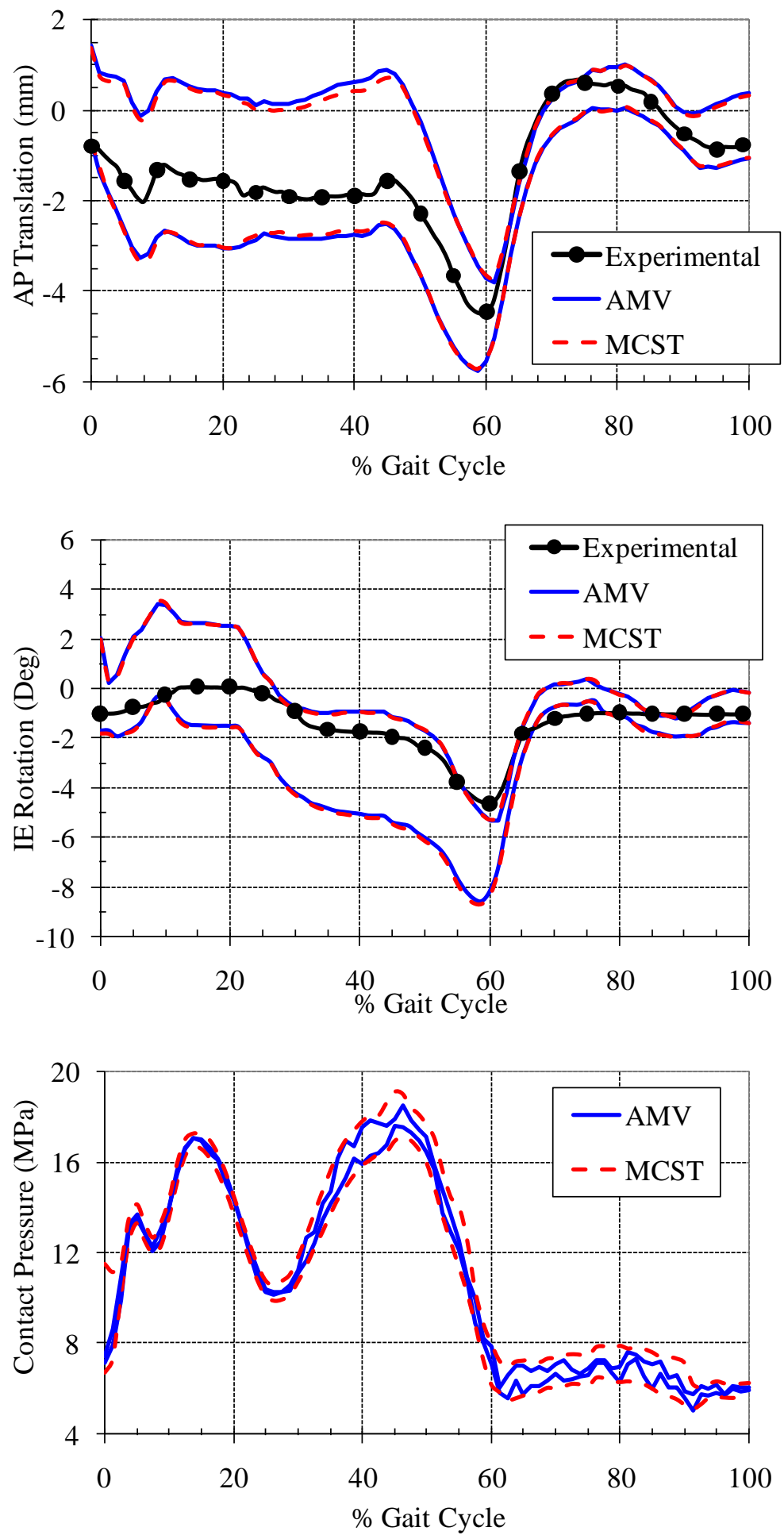

Figure 5. Comparison of predicted envelopes (1-99\%) for AMV and MCST with 1000 trials using the Nessus/Abaqus platform. a) AP translation (+anterior/-posterior), b) IE rotation (+external/-internal), and c) peak contact pressure 
Anterior Posterior Translation

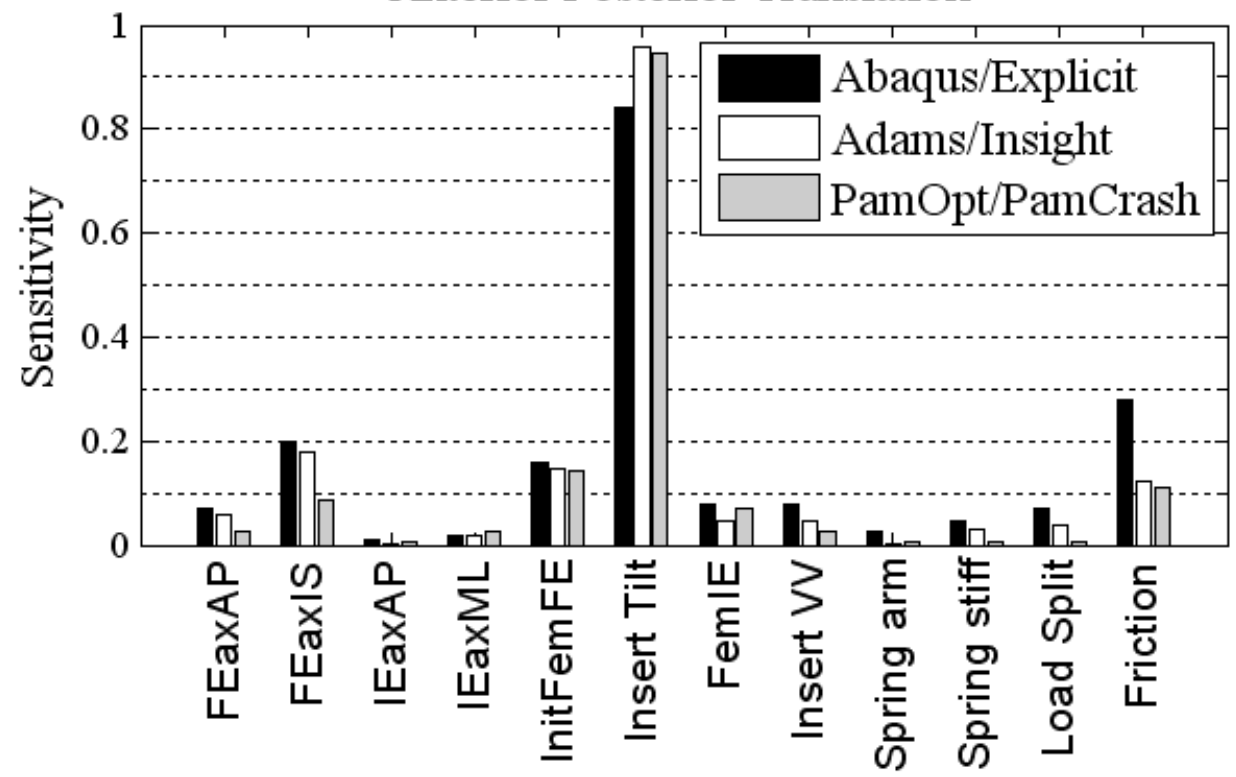

(a)

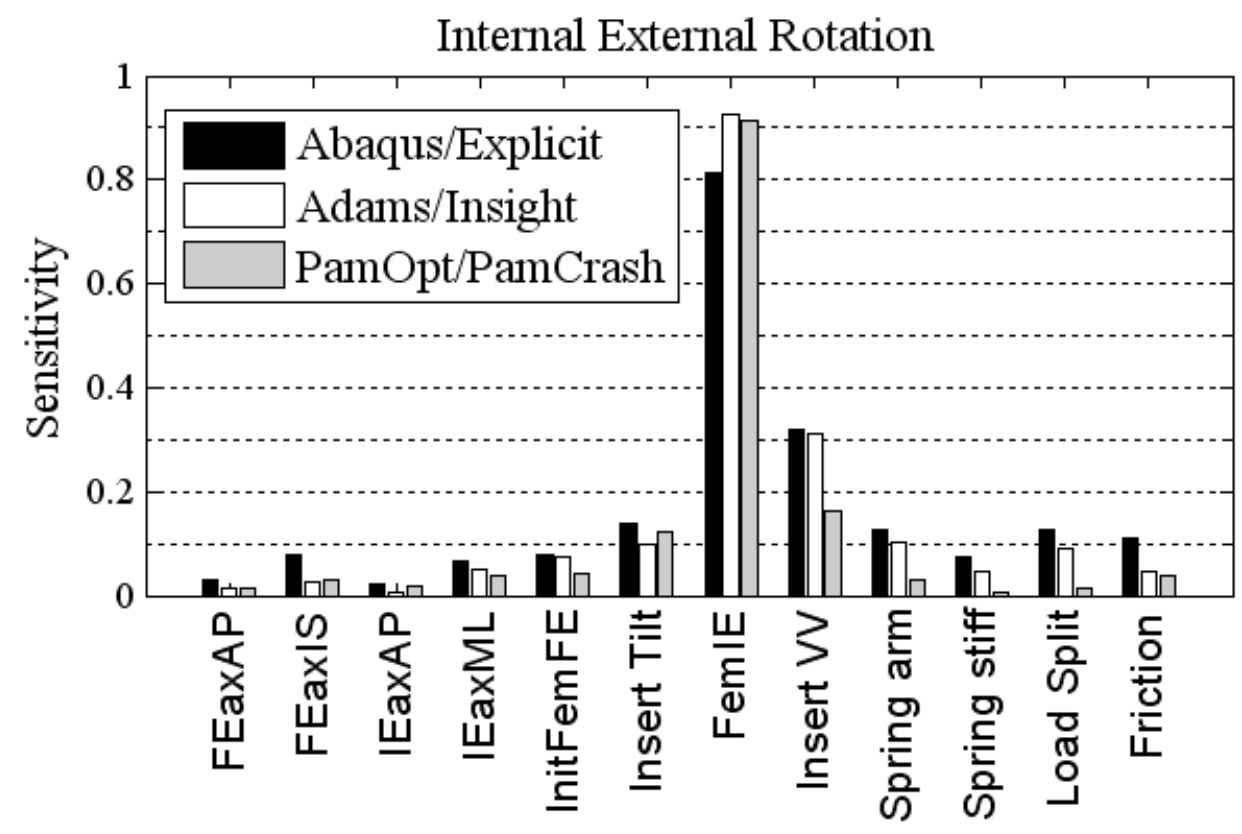

(b) 


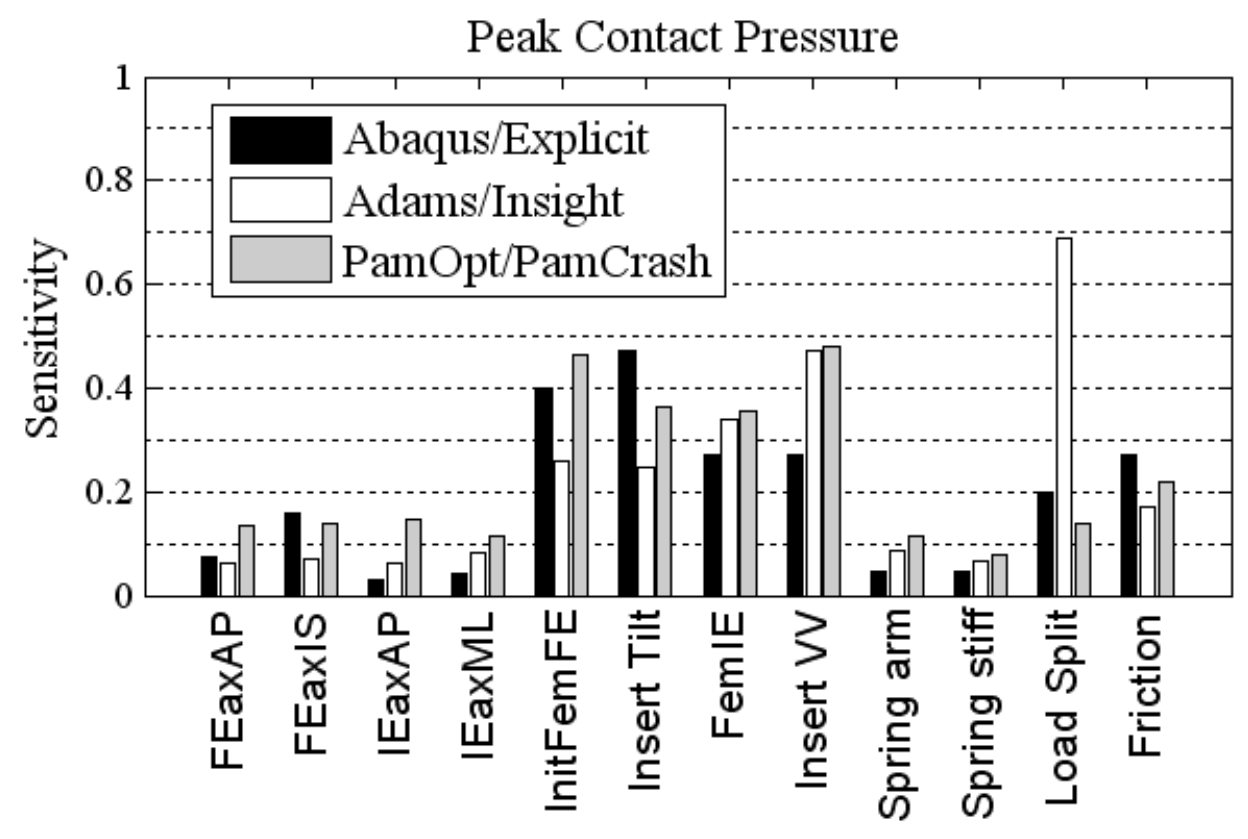

(c)

Figure 6. Sensitivity results for the various platforms represented by the averaged absolute values over the gait cycle for: a) AP translation, b) IE rotation, and c) peak contact pressure. 


\begin{tabular}{|c|c|c|}
\hline Parameter & Mean value & Variability $\sigma$ \\
\hline AP position of femoral FE axis (FEax_AP) & $0 \mathrm{~mm}$ & \multirow{4}{*}{$0.5 \mathrm{~mm}$} \\
\hline IS position of femoral FE axis (FEax_IS) & $25.4 \mathrm{~mm}$ & \\
\hline AP position of tibial IE axis (IEax_AP) & $7.62 \mathrm{~mm}$ & \\
\hline ML position of tibial IE axis (IEax_ML) & $0 \mathrm{~mm}$ & \\
\hline Initial femoral FE rotation (Init_Fem_FE) & \multirow{4}{*}{$0^{\circ}$} & \multirow{4}{*}{$1^{\circ}$} \\
\hline Initial femoral IE rotation (Fem_IE) & & \\
\hline Tilt of the tibial insert (Insert Tilt) & & \\
\hline VV position of the tibial insert (Insert_VV) & & \\
\hline ML position of spring fixation ( $\Delta \mathrm{ML})$ & $28.7 \mathrm{~mm}$ & $0.5 \mathrm{~mm}$ \\
\hline Spring stiffness $(\mathrm{K})$ & $5.21 \mathrm{~N} / \mathrm{mm}$ & $0.09 \mathrm{~N} / \mathrm{mm}$ \\
\hline ML load split (60\%-40\%) (ML_Load) & $60 \%$ & $2.5 \%$ \\
\hline Friction coefficient $(\mu)$ & 0.04 & 0.01 \\
\hline
\end{tabular}

Table 1. Input factors for probabilistic study from (Laz et al., 2006a). 


\begin{tabular}{|c|c|c|c|}
\hline Deterministic Comparison & $\begin{array}{c}\text { Anterior-Posterior } \\
\text { Translation }(\mathrm{mm})\end{array}$ & $\begin{array}{c}\text { Internal-External } \\
\text { Rotation }\left({ }^{\circ}\right)\end{array}$ & $\begin{array}{c}\text { Peak Contact } \\
\text { Pressure (MPa) }\end{array}$ \\
\hline Abaqus and experiment & 0.5 & 0.9 & N/A \\
\hline ADAMS and experiment & 0.8 & 1.3 & N/A \\
\hline PAM-CRASH and experiment & 0.6 & 0.9 & N/A \\
\hline Average & 0.6 & 1.0 & 1.1 \\
\hline ADAMS and Abaqus & 0.5 & 0.9 & 2.1 \\
\hline PAM-CRASH and Abaqus & 0.6 & 0.9 & 3.0 \\
\hline ADAMS and PAM-CRASH & 0.4 & 1.0 & 2.1 \\
\hline Average & 0.5 & 0.9 & \\
\hline
\end{tabular}

Table 2. Root mean square (RMS) differences between the various platforms. 


\begin{tabular}{|c|c|c|c|}
\hline Measure / Platform & $\begin{array}{c}\text { Anterior-Posterior } \\
\text { Translation }(\mathrm{mm}) \\
\text { Ave / Max }\end{array}$ & $\begin{array}{c}\text { Internal-External } \\
\text { Rotation }\left(^{\circ}\right. \\
\text { Ave / Max }\end{array}$ & $\begin{array}{c}\text { Peak Contact } \\
\text { Pressure (MPa) } \\
\text { Ave / Max }\end{array}$ \\
\hline \hline Nessus/Abaqus & $2.2 / 3.4$ & $2.8 / 4.5$ & $1.4 / 4.8$ \\
MCST (1000 trials) & $2.3 / 3.4$ & $2.7 / 4.3$ & $0.5 / 1.7$ \\
AMV & $2.7 / 4.1$ & $3.1 / 4.4$ & $2.5 / 3.7$ \\
\hline ADAMS/Insight & $2.7 / 4.0$ & $3.1 / 4.5$ & $1.8 / 3.4$ \\
MCST (1000 trials) & $2.9 / 4.1$ & & \\
RSM (50 trials) & $2.8 / 4.1$ & $2.8 / 4.5$ & $2.8 / 6.2$ \\
\hline PAM-OPT/ PAM-CRASH & $2.8 / 4.5$ & $2.4 / 5.7$ \\
MCST (1000 trials) & RSM (50 trials) & & \\
\hline
\end{tabular}

Table 3. Comparison of average and maximum ranges of predicted 1 and 99 percentile bounds for the various performance metrics and platforms. 